Document downloaded from:

http://hdl.handle.net/10251/38113

This paper must be cited as:

Barrachina, J.; Garrido, P.; Fogue, M.; Martínez, FJ.; Cano Escribá, JC.; Tavares De Araujo Cesariny Calafate, CM.; Manzoni, P. (2013). Road side unit deployment: a densitybased approach. IEEE Intelligent Transportation Systems Magazine. 5(3):30-39. doi:10.1109/MITS.2013.2253159.

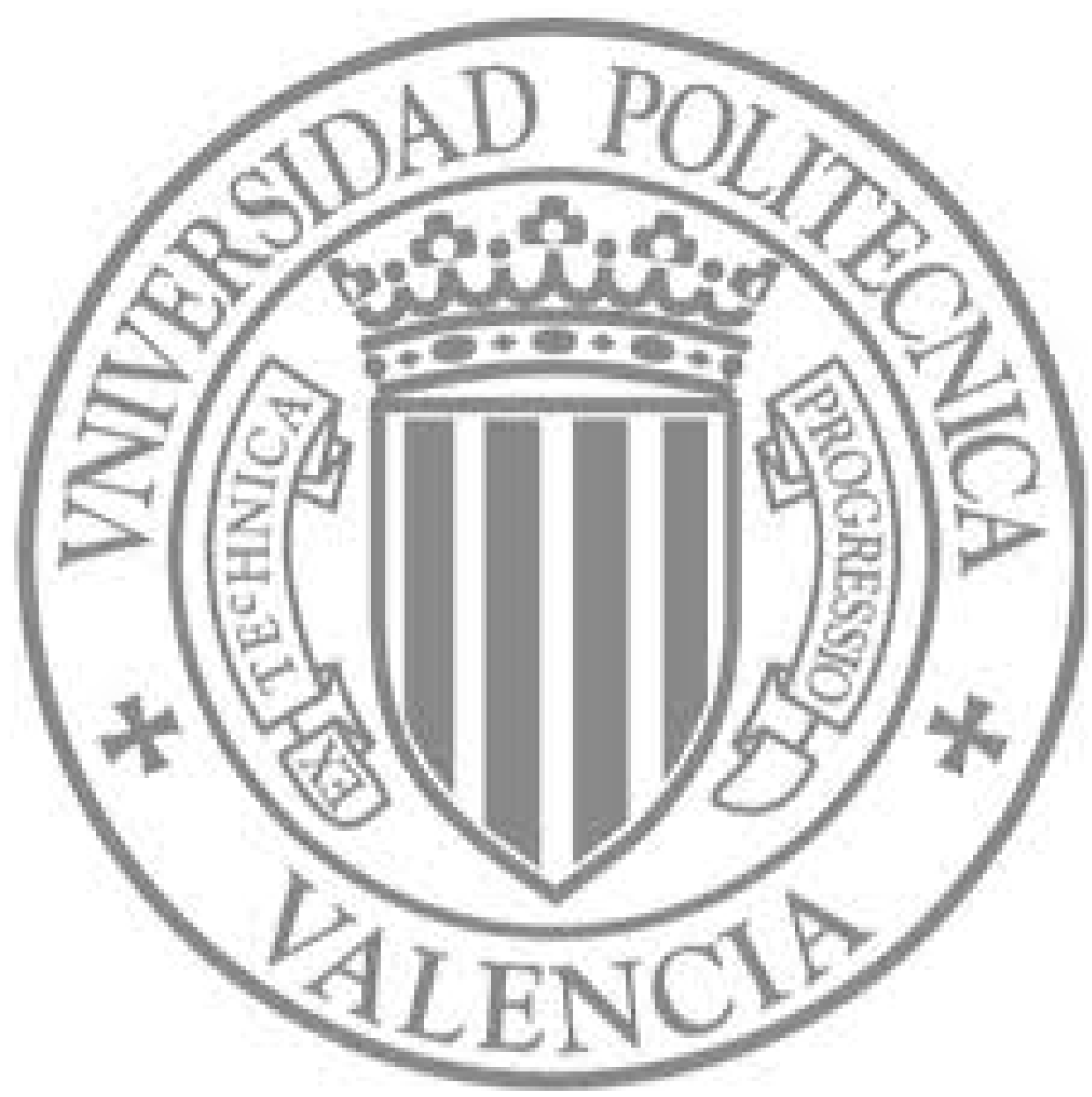

The final publication is available at

http://ieeexplore.ieee.org/xpl/articleDetails.jsp?arnumber=6565484

Copyright Institute of Electrical and Electronics Engineers (IEEE) 


\title{
Road Side Unit Deployment: A Density-Based Approach
}

\author{
Javier Barrachina, Piedad Garrido, Manuel Fogue, \\ Francisco J. Martinez \\ University of Zaragoza, Spain \\ Email: $\{$ barrachina, piedad, m.fogue, f.martinez\}@unizar.es \\ Juan-Carlos Cano, Carlos T. Calafate, Pietro Manzoni, \\ Universitat Politècnica de València, Spain \\ Email: \{jucano, calafate, pmanzoni\}@disca.upv.es
}

\begin{abstract}
Currently, the number of vehicles increases every year, raising the probability of having accidents. When an accident occurs, wireless technologies enable vehicles to share warning messages with other vehicles by using vehicle to vehicle (V2V) communications, and with the emergency services by using vehicle to infrastructure (V2I) communications. Regarding vehicle to infrastructure communications, Road Side Units (RSUs) act similarly to wireless LAN access points, and can provide communications with the infrastructure. Since RSUs are usually very expensive to install, authorities limit their number, especially in suburbs and areas of sparse population, making RSUs a precious resource in vehicular environments. In this paper, we propose a Density-based Road Side Unit deployment policy (D-RSU), specially designed to obtain an efficient system with the lowest possible cost to alert emergency services in case of an accident. Our approach is based on deploying RSUs using an inverse proportion to the expected density of vehicles. The obtained results shows how D-RSU is able to reduce the required number of RSUs, as well as the accident notification time.
\end{abstract}

\section{Index Terms}

Vehicular networks, Road Side Unit, vehicle-to-infrastructure communication, road safety.

\section{INTRODUCTION}

Nowadays, mobility requirements make transportation systems a fundamental element in our lives. Hence, transportation infrastructure investments represent a considerable fraction of the total Governments investments around the globe. Moreover, the emergence of modern Intelligent Transportation Systems (ITS) based on vehicular networks, which add wireless communication capabilities to the current vehicles, requires the deployment of efficient roadside communication infrastructures. These infrastructure assets are generally characterized by high deployment costs, and they are generally funded and managed by national governments.

In addition, the number of vehicles circulating on the roads drastically increases every year, and traffic accidents represent a serious drama in our society. Therefore, safety also acquires a special relevance when accounting for transportation systems [11]. Governments increasingly are establishing restrictive regulations to improve safety on roads, so that current roads are designed to be safer. Moreover, the automotive industry adds new safety elements inside vehicles, (e.g. airbags, stability control systems, antilock brake systems, etc.). However, the number of accidents still increases every year all over the world, being the number of fatalities also higher.

A close look at the accidents shows that many of the deaths occurred during the time between the accident and the arrival of medical assistance. The so called golden hour after a car crash is the time within which medical or surgical intervention by a specialized trauma team has the greatest chance of saving lives. If more than 60 minutes have elapsed by the time the patient arrives to the operating table, the chances of survival fall sharply. Typical arrival of medical help takes about 15 minutes, but initial access and treatment only starts 25 minutes after the accident. Transportation of the injured to the hospital usually takes place only 50 minutes later. Therefore, time is critical to the survival of the injured in a severe incident. Hence, any technology capable of providing a fast and efficient rescue operation after a traffic accident will increase the probability of survival of the injured, and so reducing the injury severity.

Wireless technologies, through vehicular networks, enable peer-to-peer mobile communications among vehicles (V2V), as well as communications between vehicles and infrastructures (V2I) [13], which allow to avoid collisions among vehicles [14]. In addition, using these technologies, crashed vehicles are able to alert nearby vehicles, as well as to notify emergency services when an accident occurs.

The combination of V2V and V2I communications can propel our communication capabilities. Regarding traffic safety, by adding infrastructure to vehicular networks, two benefits are provided: (i) infrastructure can provide Internet access to vehicles, allowing them to communicate with the emergency services immediately, thereby reducing notification times in case of an 
accident, and (ii) infrastructure access points can rebroadcast messages delivered by vehicles in low vehicle density scenarios (allowing messages to arrive to more vehicles).

A Road Side Unit (RSU) is a communication node installed within the infrastructure. Therefore, the capacity of vehicles to communicate with an infrastructure depends on the number and radio coverage of existing RSUs in the nearby area. However, Roadside Units are usually very expensive to install. Hence, authorities tend to limit their number, especially in suburbs and sparse population areas, making RSUs a precious resource in vehicular environments.

In this work, we propose a density-based approach for RSU deployment in urban scenarios and compare it with other alternative deployment policies to obtain an efficient system. Our aim is to reduce the deployment cost by minimizing the required number of RSUs, as well as to reduce the warning notification time (i.e., the time required to notify emergency services and other vehicles). Specifically, we tested these deployment policies by simulating a urban scenario where vehicles want to alert both the emergency services and the nearby vehicles.

This paper is organized as follows: Section II reviews the related work regarding vehicular networks using infrastructure. In Section III we present three different RSU deployment policies (i.e., Minimum Cost, Uniform Mesh, and D-RSU). Section IV introduces the simulation environment to assess our proposal. Section V shows the obtained results. Finally, Section VI concludes this paper.

\section{RELATED WORK}

Vehicular networks have been studied for several years but, although there are many studies regarding V2V communications, only a few focused on V2I communications, too.

Regarding V2I communications, Wu et al. [16] proposed a novel mechanism, called Distributed Sorting Mechanism (DSM), to improve the efficiency of communication between vehicles and RSUs. In their work, every vehicle can individually calculate its own priority of communication, in order to reduce the time required to compete and obtain the channel. Authors also consider that vehicles moving away from the coverage of communication can appropriately adjust their priorities.

Mershad et al. [12] studied how to exploit RSUs to route packets between any source and destination in vehicular networks. They evaluated the RSU backbone routing performance via simulation, and compared their scheme to existing solutions, proving the feasibility and efficiency of their scheme in terms of query delay, packet success delivery ratio, and total generated traffic.

Fogue et al. [4] presented eNOTIFY, a novel proposal based on the combination of V2V and V2I communication capabilities, designed to improve the responsiveness of emergency services by reducing the required time to rescue the passengers involved in a car accident, and automatically managing and optimizing the medical and rescue needed resources.

Furthermore, other authors studied how to place RSUs. Lochert et al. [8] proposed a genetic algorithm which was able to identify good positions for static RSUs in order to cope with the highly partitioned nature of a vehicular network in an early deployment stage for the city of Brunswick. They defended a tailored toolchain to optimize the placement with respect to an application-centric objective function. However, for all their simulations, they only used a reduced average equipment density of 0.25 equipped vehicles per radio range.

Kchiche and Kamoun [5] provided an analysis of the infrastructure deployment problem. Authors considered that the use of RSUs becomes essential for communications among vehicles in low density situations. They proved that centrality and equidistance are key factors for optimizing end-to-end delays and ensuring stable performances. However, their simulations were made only using a density of 200 vehicles in a total route-length of about $75 \mathrm{~km}$.

Lee and Kim [7] proposed a roadside unit placement scheme for vehicular networks, aiming at improving connectivity and reducing the disconnection interval for the given number of roadside units, the transmission range, and the overlap ratio on the road network of Jeju island. Performance measurement results obtained using the real-life movement history data in Jeju city showed that about $72.5 \%$ of connectivity can be achieved when deploying 1,000 RSUs, being the transmission range 300 $\mathrm{m}$, while the disconnection time is mostly kept below 10 seconds.

To the best of our knowledge, few proposals studied the RSU deployment problem, optimizing the placement of RSUs to maximize performance and reduce the deployment cost. Furthermore, these proposals have been tested in very simple scenarios with very specific traffic densities.

\section{RSUS DePLOYMENT POLICIES}

Roadside Units are usually expensive to install. Therefore, authorities limit their number, especially in suburbs and areas of sparse population, making RSUs a precious resource in vehicular environments. Moreover, given the current economic situation, authorities and transport agencies tend to reduce the infrastructure investments related to transportation systems.

Therefore, the selected deployment policy is of utmost importance when adding infrastructure for vehicular networks. Authorities could place RSUs in a homogeneous way (uniformly) trying to maximize the coverage area, or following a non uniform deployment approach (e.g., grouping RSUs in specific parts of an area) trying to reduce the deployment cost.

Similarly to the traffic lights or the traffic luminous panels, before deploying RSUs, authorities should make a preliminary study which involves gathering important data (regarding the economic impact, or the number of potential users) to decide where and how to deploy the infrastructure stations. We consider that Governments should also pay particular attention to 


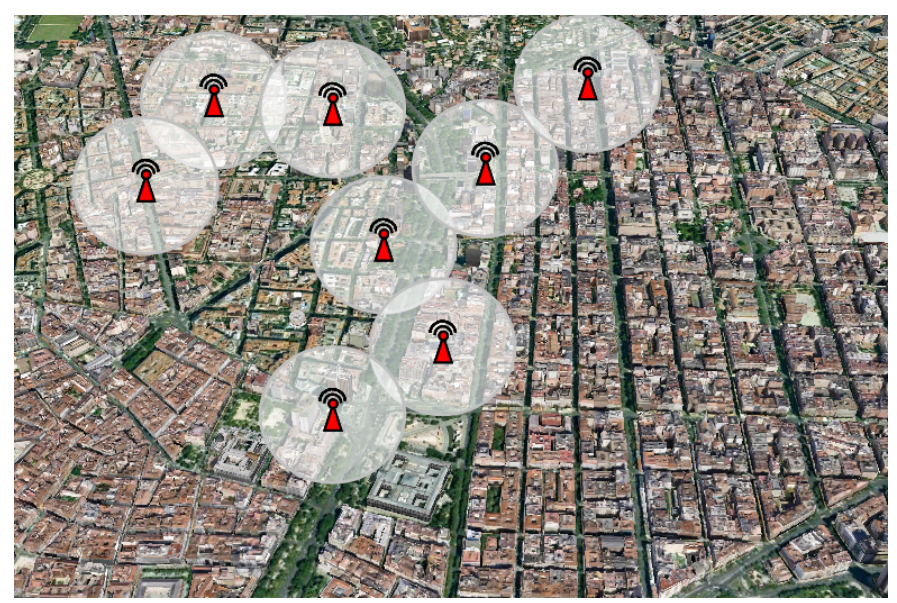

Fig. 1. Example of 8 RSUs deployed following the Minimum Cost deployment policy.

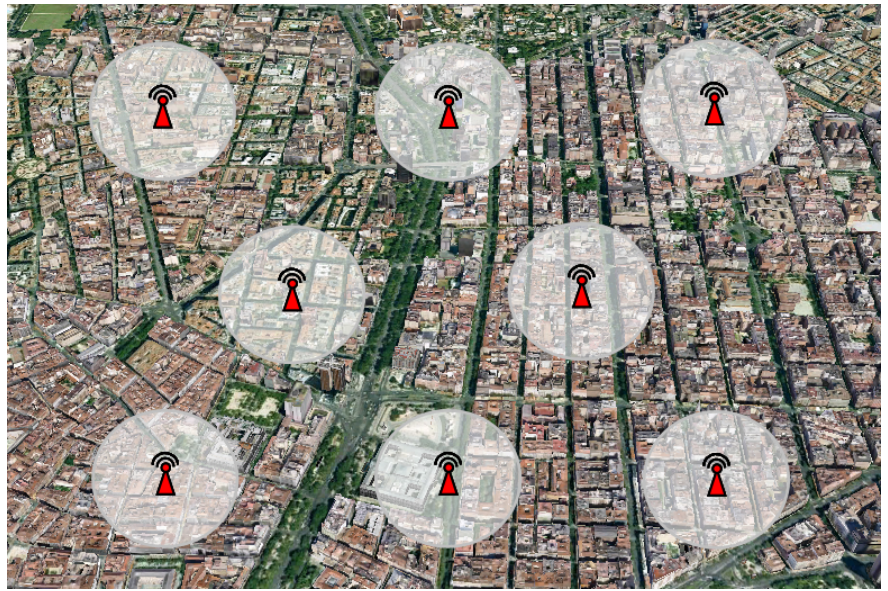

Fig. 2. Example of 8 RSUs deployed following the Uniform Mesh deployment policy.

accounting for the expected density of vehicles in order to optimize the infrastructure deployments, thereby reducing the economic cost, without reducing the time required by vehicles to get access to RSUs in case of emergency.

In the following Subsections we present in detail three different RSUs deployment policies: (i) the Minimum Cost, (ii) the Uniform Mesh, and (iii) the Downtown-based (D-RSU).

\section{A. Minimum Cost Deployment Policy}

In the Minimum Cost deployment policy, we consider that authorities only account for reducing the economic cost of the infrastructure deployment, without considering whether RSUs will be placed to maximize the coverage area, or not. This policy consists on distributing RSUs with the minimum possible cost. In real environments, this policy would place the RSUs in locations that already have Internet access, or where their installation is easy, regardless of their position in the map. Figure 1 shows an example of this deployment policy.

The total cost of this policy is the lowest, since authorities can place RSUs in sites where there are already Internet access (e.g., nearby government buildings, traffic luminous panels, etc.). However, this deployment policy can provoke that some areas remain isolated, without infrastructure coverage.

\section{B. Uniform Mesh Deployment Policy}

The Uniform Mesh deployment policy consists on distributing RSUs uniformly on the map, regardless of the expected average traffic density, or the roadmap topology.

The advantage of this deployment policy is that it achieves a more uniform coverage area since the distance between RSUs is basically the same, preventing RSUs to be positioned too closely, or too dispersed. An example of this deployment policy is shown in Figure 2.

The Uniform Mesh policy tends to reduce the probability of having shadow areas in the map, where vehicles can remain isolated (without the possibility of alert emergency services in case of accident). 


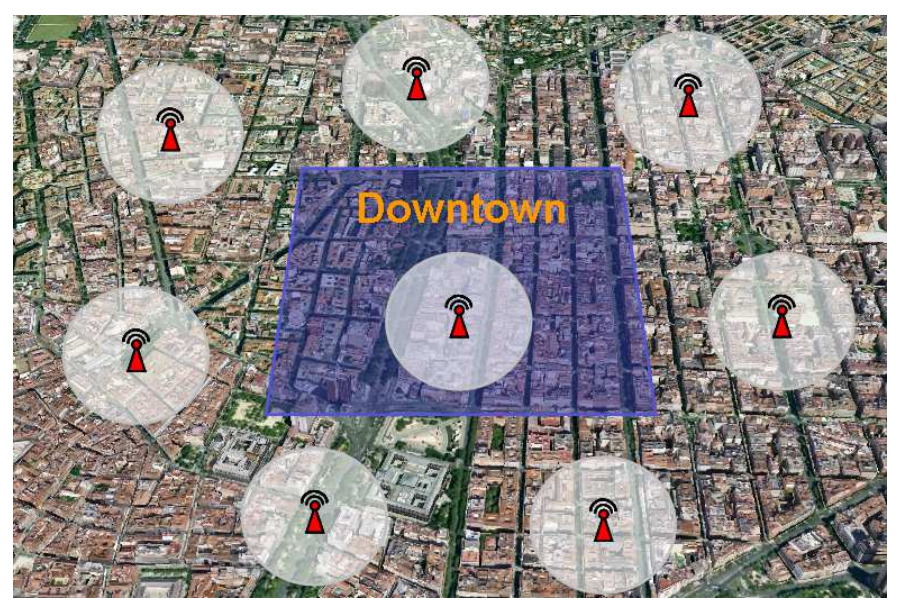

Fig. 3. Example of 8 RSUs deployed following the D-RSU deployment policy.

TABLE I

MAIN FEATURES OF THE SELECTED MAP

\begin{tabular}{|l|c|}
\hline \hline Selected city map & Madrid (Spain) \\
Total streets & 1387 \\
Total junctions & 715 \\
Avg. street length & $83.08 \mathrm{~m}$ \\
Avg. lanes/street & 1.27 \\
\hline \hline
\end{tabular}

This deployment policy has a higher cost than the previous one, because authorities have to provide Internet access in all the places where the RSUs are positioned.

\section{D-RSU Deployment Policy}

In vehicular scenarios, traffic is not uniformly distributed; there are zones that usually have a higher vehicle density. For example, in the cities, these zones are usually located in the downtown area, business areas, or industrial areas, where the higher density of vehicles makes them move more slowly than in the outskirts, but also increases the number of potential nodes of a vehicular network based on $\mathrm{V} 2 \mathrm{~V}$ communications.

In the D-RSU deployment polity, RSUs are placed using an inverse proportion to the expected density (i.e., more resources must be deployed in areas where less number of vehicles are usually expected, and authorities must deploy less RSUs in areas which are characterized by a high density of vehicles). For example, if authorities have in mind to deploy a total number of 100 RSUs in a city, and they consider that the expected traffic density in an specific area of this city is about $60 \%$ of the vehicles, according to the D-RSU deployment policy, they should install $40 \%$ of RSUs there, and the rest of the RSUs should be deployed in the rest of the area.

This deployment policy allows vehicles located in less dense areas to have a better Internet access capabilities thanks to the higher density of deployed RSUs. In contrast, we consider that areas which support a high density of vehicles do not require a high number of RSU, since V2V communications can complement the infrastructure (i.e., high reliable V2V scenarios do not require a great infrastructure without reduce performance). Figure 3 shows an example of this kind of deployment policy.

Similar to the Uniform Mesh deployment policy, D-RSU has a higher economical cost than the Minimum Cost deployment policy, since authorities need to provide Internet access in all the places where the RSUs are positioned.

\section{Simulation EnVironment}

Simulations were done using the ns-2 simulator, where the PHY and MAC layers have been modified to follow the IEEE 802.11p standard, which defines enhancements to the 802.11 required to support Intelligent Transportation System (ITS) applications. We assume that all the nodes of our network have two different interfaces: (i) an IEEE 802.11n interface tuned at the frequency of $2.4 \mathrm{GHz}$ for V2I communications, and (ii) an IEEE 802.11p interface tuned at the frequency of $5 \mathrm{GHz}$ for $\mathrm{V} 2 \mathrm{~V}$ communications.

In our simulations, vehicles can operate in two different modes: (a) warning, and (b) normal. Vehicles in warning mode inform other vehicles about their status by sending warning messages periodically (every second). These messages have the highest priority at the MAC layer. Normal mode vehicles enable the diffusion of these warning packets and, every second they also send beacons with information such as their positions, speed, etc. These periodic messages have lower priority than warning messages and are not propagated by other vehicles. As for the RSUs, they mainly provide Internet access to vehicles, but they also enable the diffusion of the warning messages. 
TABLE II

PARAMETER VALUES FOR THE Simulations

\begin{tabular}{|l|l|}
\hline \hline Parameter & Value \\
\hline density of vehicles $\left(\mathrm{veh} . / \mathrm{km}^{2}\right)$ & $25,50,75,100,125$, and 150 \\
number of RSUs & $1,2,4,8$, and 16 \\
simulated city & Madrid \\
simulated area & $2000 \mathrm{~m} \times 2000 \mathrm{~m}$ \\
number of crashed vehicles & 2 \\
downtown size & $1000 \mathrm{~m} \times 1000 \mathrm{~m}$ \\
downtown probability & 0.7 \\
warning message size & 13 and $18 \mathrm{~KB}$ \\
packets sent by vehicles & 1 per second \\
warning message priority & $A C 3$ \\
normal message priority & $A C 1$ \\
mobility generator & $C 4 R$ [2] \\
mobility models & Krauss [6] and Downtown [9] \\
MAC/PHY & $802.11 \mathrm{p}$ \\
radio propagation model & $\mathrm{RAV}[10]$ \\
maximum transmission range & $400 \mathrm{~m}$ \\
broadcast storm reduction scheme & eMDR [3] \\
\hline \hline
\end{tabular}

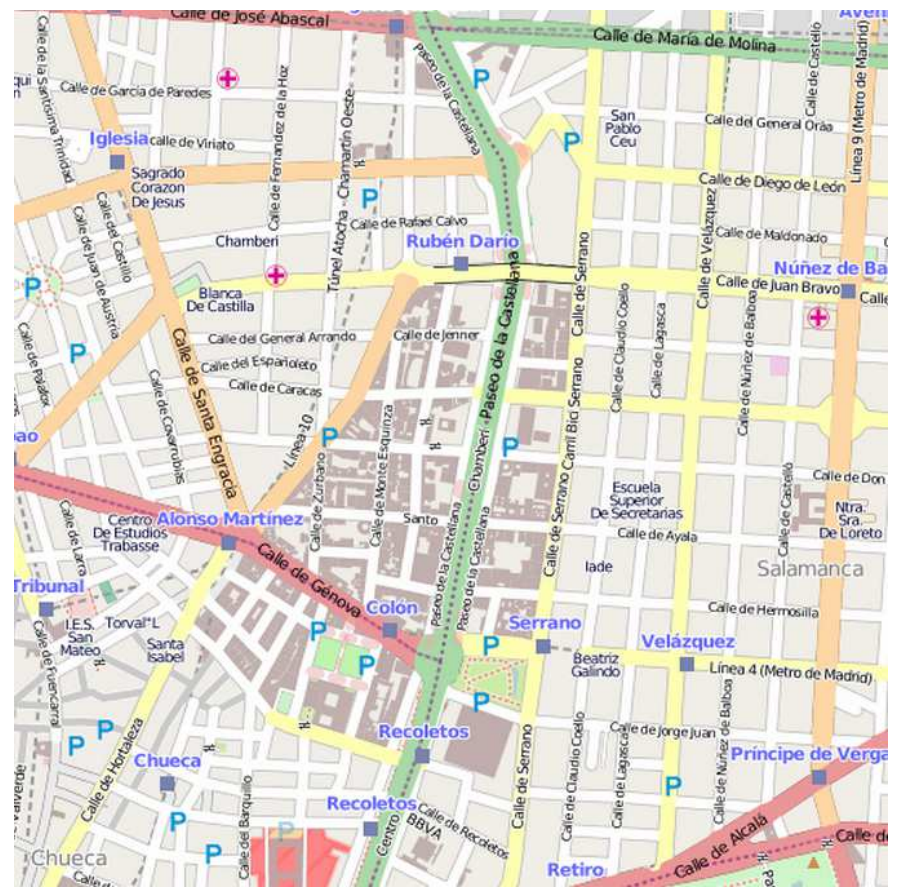

Fig. 4. Fragment of the city of Madrid (Spain) used in our simulations.

To increase the realism of our simulations, we use CityMob for Roadmaps (C4R $)^{1}$ [2], a mobility generator based on SUMO. C4R includes all the original characteristics from SUMO (collision-free vehicle movements, multi-lane streets, etc.). In addition, it is able to define attraction and repulsion points which simulate areas with different vehicle densities in real cities [9].

Our simulations use a scenario of $4 \mathrm{~km}^{2}$, obtained from the city of Madrid (Spain). Figure 4 shows the topology of the map, and Table I includes the main features of the selected city.

Since our proposal is focused on the use of an infrastructure to notify emergency services when an accident occurs, the warning messages exchanged between vehicles and RSUs are built according the Vehicular Accident Ontology (VEACON) [1], specially designed for sharing and reusing knowledge about the vehicles involved in road accidents. We simulated several front impact scenarios with two cars involved. The first vehicle is a family car with two occupants, and expressing all the information required produces a message of 13 KBytes. The second car is a minivan with eight occupants, which required up to $18 \mathrm{KBytes}$ to code the data for all passengers. Each simulation run lasted for 300 seconds. All results represent an average of 20 executions with different scenarios (maximum error of $10 \%$ with a degree of confidence of $90 \%$ ). Table II shows the parameters used in the simulations. 
TABLE III

Simulation Results for the Uniform Mesh Deployment Policy When Varying the Density of Vehicles and RSUs

\begin{tabular}{|c|c||c|c||c|c|}
\hline \hline Vehicles/km ${ }^{2}$ & RSUs & Min. notif. time (s) & Max. notif. time (s) & Avg. notif. time (s) & Accident notif. (\%) \\
\hline \hline \multirow{5}{*}{25} & 1 & 0.476 & 31.003 & 13.974 & 75 \\
\cline { 2 - 6 } & 2 & 0.563 & 30.422 & 12.389 & 85 \\
\cline { 2 - 6 } & 4 & 0.676 & 26.701 & 9.650 & 85 \\
\cline { 2 - 6 } & 8 & 0.273 & 11.257 & 3.222 & 95 \\
\hline \hline \multirow{5}{*}{50} & 16 & 0.230 & 15.753 & 2.935 & 100 \\
\cline { 2 - 6 } & 2 & 0.827 & 33.125 & 9.937 & 100 \\
\cline { 2 - 6 } & 4 & 0.476 & 33.919 & 8.209 & 100 \\
\cline { 2 - 6 } & 8 & 0.363 & 12.958 & 3.471 & 100 \\
\cline { 2 - 6 } & 16 & 0.125 & 10.949 & 2.640 & 100 \\
\hline \hline \multirow{5}{*}{75} & 1 & 0.333 & 21.281 & 1.922 & 100 \\
\cline { 2 - 6 } & 2 & 0.777 & 9.546 & 4.683 & 100 \\
\cline { 2 - 6 } & 4 & 0.543 & 13.053 & 4.453 & 100 \\
\cline { 2 - 6 } & 8 & 0.852 & 6.782 & 3.000 & 100 \\
\hline \hline \multirow{5}{*}{100} & 16 & 0.272 & 10.111 & 1.931 & 100 \\
\cline { 2 - 6 } & 2 & 0.241 & 10.460 & 1.328 & 100 \\
\cline { 2 - 6 } & 4 & 0.999 & 11.594 & 3.551 & 100 \\
\cline { 2 - 6 } & 8 & 0.564 & 11.833 & 2.983 & 100 \\
\cline { 2 - 6 } & 16 & 0.550 & 11.604 & 3.078 & 100 \\
\hline
\end{tabular}

\section{Simulations Results}

In this section, we first study the required number of RSUs per square kilometer to have a feasible warning notification system, where crashed vehicles can properly alert the emergency services, as soon as possible. Then, we also show the impact of the RSUs deployment policy in the obtained results when varying the number of RSUs, and the density of vehicles in the scenario. Our aim is to determine which policy fits better in the studied conditions.

In our simulations, we measure notification times (the minimum, the maximum, and the average time that a warning message requires to reach an RSU), and the percentage of accidents that have been successfully notified (i.e., that have already reached an RSU, thereby considering that emergency services have been correctly notified).

\section{A. Study of Required Number of RSUs}

Table III shows the obtained results for the Uniform Mesh deployment policy when varying the number of RSUs deployed $(1,2,4,8$, and 16$)$, and the density of vehicles $(25,50,75,100$, and 125) in the selected scenario.

As shown, when the density becomes very small (i.e., 25 vehicles $/ \mathrm{km}^{2}$, which is clearly infrequent in urban scenarios), and there are eight or less RSUs (two or less RSUs per square kilometer), about 5-25\% of the total accidents are not correctly notified to the emergency services (i.e., no warning message effectively reaches an RSU). However, when increasing the density of vehicles $\left(\geq 50\right.$ vehicles $\left./ \mathrm{km}^{2}\right)$, all the accidents are correctly notified, making it possible to provide precise information about the incident to reduce the response time of emergency services, thereby improving the assistance to people injured.

Regarding the average notification time, it ranges from 0.946 seconds (100 vehicles $/ \mathrm{km}^{2}$ and 16 RSUs) to 13.974 seconds (25 vehicles $/ \mathrm{km}^{2}$ and $1 \mathrm{RSU}$ ). As expected, the system requires less time to reach an RSU when increasing the number of RSUs, although we observe that in high density situations $\left(125\right.$ vehicles $\left./ \mathrm{km}^{2}\right)$, specifically when 8 and 16 RSUs are deployed, the system requires more time to reach an RSU than in scenarios where 100 vehicles $/ \mathrm{km}^{2}$ are simulated. This could be explained due to redundancy, contention, and packet collisions caused by simultaneous forwarding (usually known as the broadcast storm problem [15]).

Since we are interested in safety issues, it is extremely important to be sure that all the accidents will be correctly notified to the emergency services. Hence, according to the obtained results, we consider that authorities must deploy at least four RSUs per square kilometer (16 RSUs in our example).

\section{B. Performance of the Different RSUs Deployment Policies}

Table IV shows the obtained results for the studied deployment policies, when varying the density of vehicles $(25,50,75$, 100, 125 and 150), and the number of RSUs deployed (8 and 16) in the studied scenario. For each deployment policy, we show the minimum, the maximum, and the average notification times (i.e., the time required for a warning message to reach an RSU), as well as the percentage of successfully accident notifications via the infrastructure. 
TABLE IV

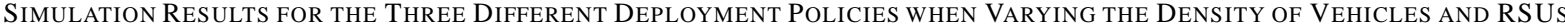

\begin{tabular}{|c|c|c|c|c|c|c|c|c|c|c|c|c|c|}
\hline \multirow{2}{*}{\multicolumn{2}{|c|}{$\begin{array}{c}\text { Vehicles } / \mathbf{k m}^{2} \\
\text { RSUs }\end{array}$}} & \multicolumn{2}{|c|}{25} & \multicolumn{2}{|c|}{50} & \multicolumn{2}{|c|}{75} & \multicolumn{2}{|c|}{100} & \multicolumn{2}{|c|}{125} & \multicolumn{2}{|c|}{150} \\
\hline & & 8 & 16 & 8 & 16 & 8 & 16 & 8 & 16 & 8 & 16 & 8 & 16 \\
\hline \multirow{3}{*}{ Minimum Cost } & Min. notif. time (s) & 0.394 & 0.293 & 0.228 & 0.211 & 0.460 & 0.520 & 0.308 & 0.456 & 0.390 & 0.360 & 0.306 & 0.356 \\
\hline & Max. notif. time (s) & 11.366 & 11.238 & 21.479 & 21.155 & 6.150 & 6.093 & 2.917 & 3.449 & 6.111 & 6.628 & 5.717 & 4.116 \\
\hline & Accident notif. (\%) & 95 & 95 & 100 & 100 & 100 & 100 & 100 & 100 & 100 & 100 & 100 & 100 \\
\hline \multirow{4}{*}{ Uniform Mesh } & Min. notif. time (s) & 0.273 & 0.230 & 0.125 & 0.333 & 0.272 & 0.241 & 0.265 & 0.295 & 0.360 & 0.272 & 0.302 & 0.228 \\
\hline & Max. notif. time (s) & 11.257 & 15.753 & 10.949 & 21.281 & 10.111 & 10.460 & 1.762 & 1.712 & 6.112 & 5.751 & 6.207 & 3.736 \\
\hline & Avg. notif. time (s) & 3.222 & 2.935 & 2.640 & 1.922 & 1.931 & 1.328 & 1.081 & 0.946 & 1.581 & 1.433 & 1.551 & 1.227 \\
\hline & Accident notif.(\%) & 95 & 100 & 100 & 100 & 100 & 100 & 100 & 100 & 100 & 100 & 100 & 100 \\
\hline \multirow{4}{*}{ D-RSU } & Min. notif. time (s) & 0.234 & 0.268 & 0.238 & 0.197 & 0.400 & 0.471 & 0.480 & 0.372 & 0.381 & 0.190 & 0.385 & 0.251 \\
\hline & Max. notif. time (s) & 11.161 & 11.040 & 17.400 & 20.516 & 6.282 & 6.263 & 1.832 & 2.061 & 1.723 & 1.800 & 3.459 & 3.339 \\
\hline & Avg. notif. time (s) & 3.268 & 3.333 & 3.491 & 3.071 & 1.609 & 1.091 & 0.783 & 0.771 & 0.720 & 0.688 & 1.145 & 1.100 \\
\hline & Accident notif. (\%) & 95 & 95 & 100 & 100 & 100 & 100 & 100 & 100 & 100 & 100 & 100 & 100 \\
\hline
\end{tabular}

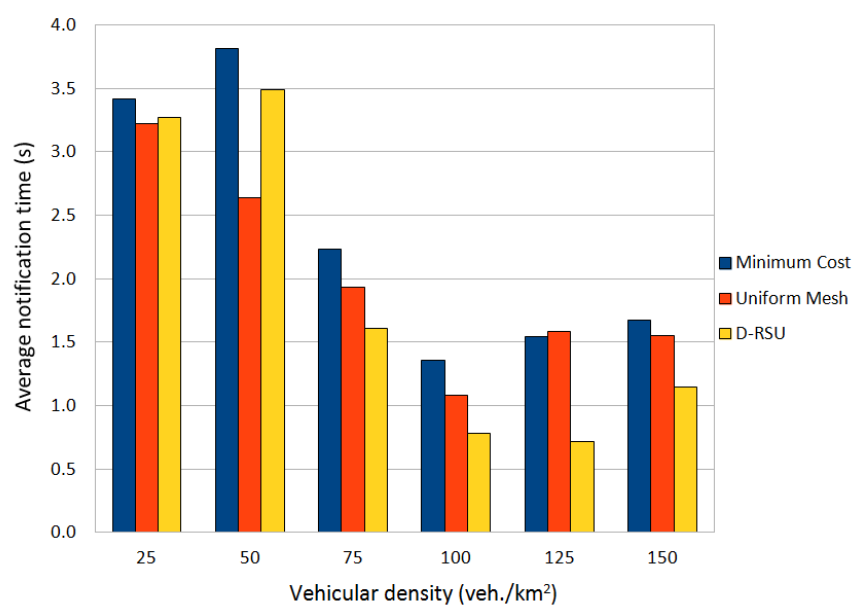

Fig. 5. Comparison of the average notification time for the studied deployment policies when using 8 RSUs.

As shown in Table IV, the warning notification system works well in all the different scenarios, since all the accidents are correctly notified to the emergency services (i.e., at least a warning message reaches an RSU). Only when the density becomes very small (25 vehicles $/ \mathrm{km}^{2}$ ), some accidents (only 5\% of the amount total) are not reported to the emergency services.

Regarding the average notification time, Figures 5 and 6 graphically depict that, in small density scenarios $\left(\leq 50 \mathrm{vehicles} / \mathrm{km}^{2}\right)$, the Uniform Mesh deployment policy yields better results than the other policies. When simulating 16 RSUs, it reduces the average notification time compared to the Minimum Cost and the D-RSU (up to $44.58 \%$ and $37.41 \%$, respectively). The Uniform Mesh policy works better in small densities, since RSUs are uniformly deployed in the map, increasing the probability that isolated vehicles have a nearby RSU. However, in higher density scenarios $\left(\geq 75\right.$ vehicles $\left./ \mathrm{km}^{2}\right)$, our D-RSU deployment policy yields better performance results, since it requires less time to inform emergency services when an accident occurs. It reduces the average notification time up to $54.16 \%$ and $51.99 \%$, compared to the Minimum Cost and to the Uniform Mesh, respectively.

These results suggest us that using the Minimum Cost approach is not a good idea, since accidents must be correctly notified in the minimum possible time.

Finally, Figure 7 shows a comparison between the D-RSU with only 8 RSUs, and the Uniform Mesh with 16 RSUs. As shown, in high density scenarios (when more than 100 vehicles per $\mathrm{km}^{2}$ are simulated), D-RSU achieves better notification times even if half of RSUs have been deployed. This shows that using D-RSU allows to severely reduce the overall cost of deploying the infrastructure, without losing performance.

\section{Our Proposed Deployment Algorithm}

Based on the obtained results, we propose the RSU deployment algorithm showed in Algorithm 1. As shown, according to Figure $8 \mathrm{a}$, in scenarios where vehicle density is lower than 70.75 vehicles per $\mathrm{km}^{2}$, the algorithm applies the Uniform Mesh deployment policy. Instead, if density is between 70.75 and 91 vehicles per $\mathrm{km}^{2}$, the D-RSU deployment policy is applied. Finally, if traffic density exceeds 91 vehicles per $\mathrm{km}^{2}$ (according to Figure 8b), our algorithm also applies the D-RSU deployment policy, but the number of required RSUs is reduced to a half with a similar performance. 


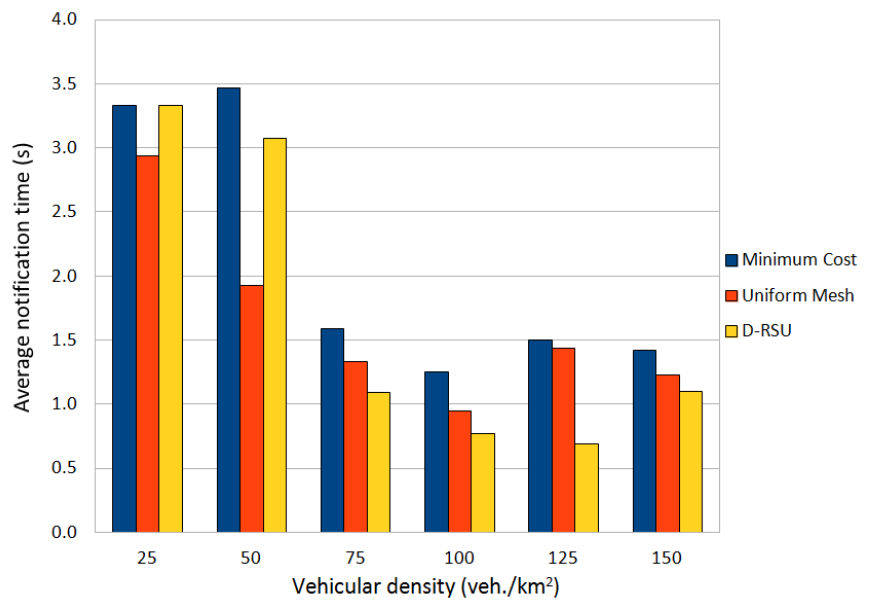

Fig. 6. Comparison of the average notification time for the studied deployment policies when using 16 RSUs.

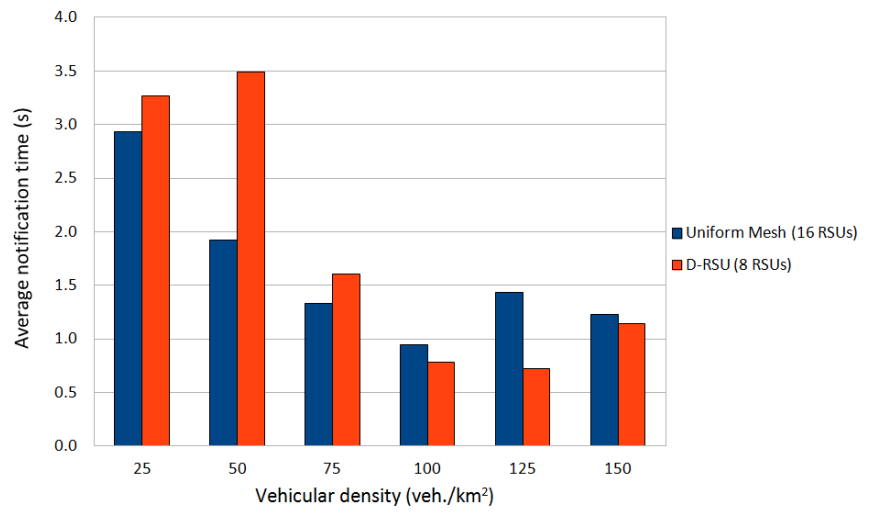

Fig. 7. Comparison between the Uniform Mesh using 16 RSUs and D-RSU using 8 RSUs.

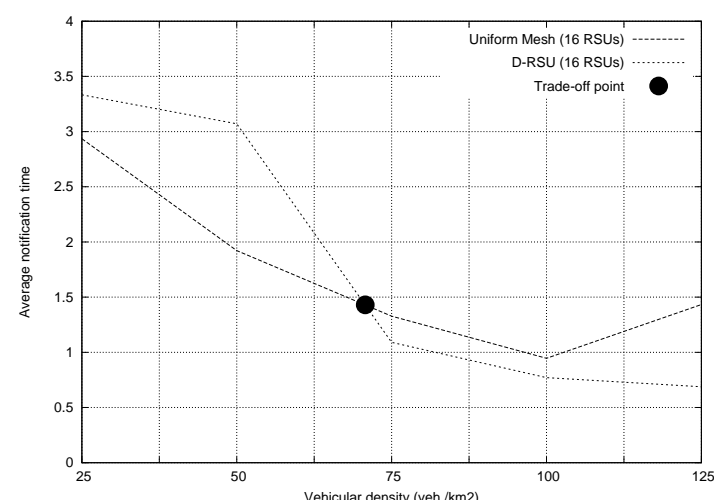

(a)

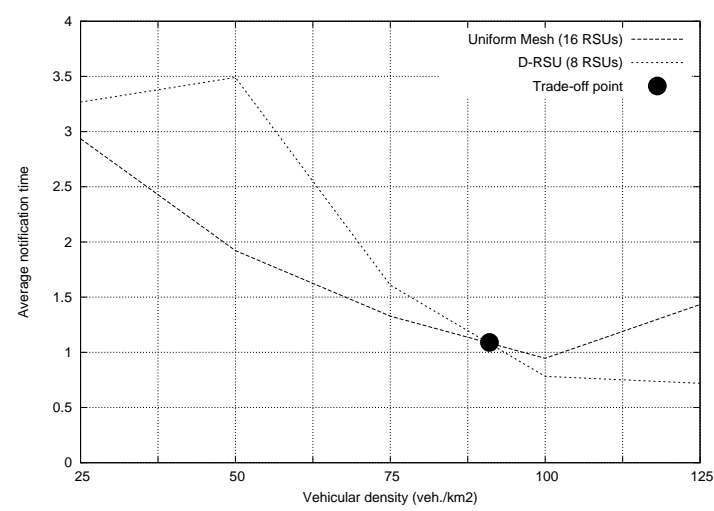

(b)

Fig. 8. Best deployment policy when the vehicular density is greater or equal than: (a) 70.75 vehicles per $\mathrm{km}^{2}$, and (b) 91 vehicles per $\mathrm{km}^{2}$. 


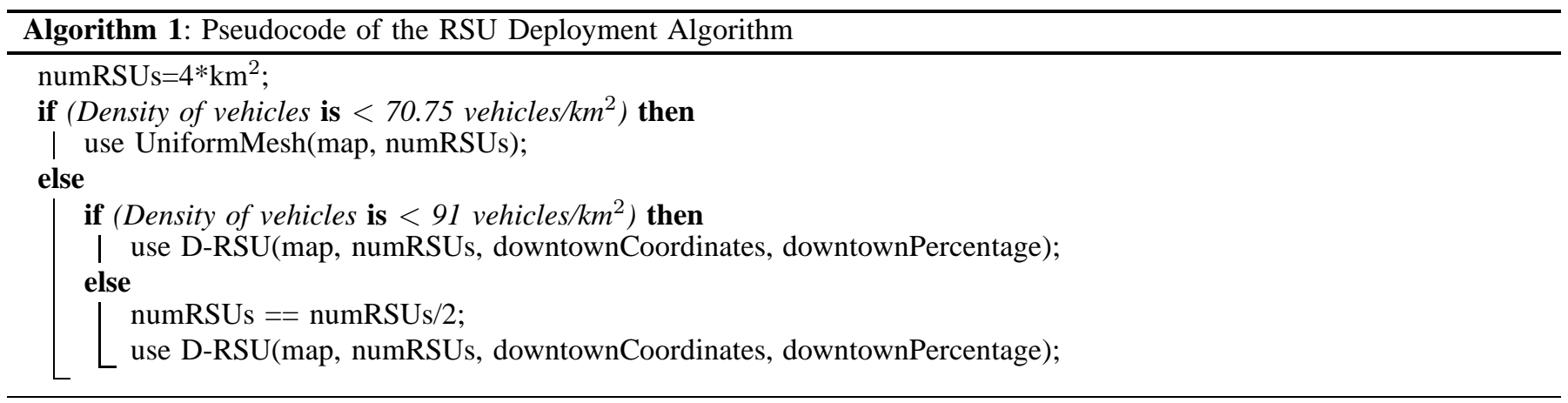

\section{CONCLUSIONS}

In this paper we present D-RSU, a density-based approach for Road Side Unit deployment in urban scenarios. D-RSU takes into account that the density of vehicles is not uniformly distributed in vehicular scenarios. D-RSU consists on placing more RSUs in areas with lower vehicle densities. Specifically, RSUs will be placed according the inverse proportion to the expected density. This distribution allows that vehicles driving in less dense areas have better Internet access by increasing the number of nearby available RSUs, whereas in areas with high density of vehicles, V2V communications can complement the infrastructure to provide a reliable warning notification system without reducing its performance.

To assess our proposal, we compare D-RSU with two other deployment policies: the Minimum Cost, and the Uniform Mesh. Simulations show that the Uniform Mesh deployment policy fits better for areas in which less than 70.75 vehicles per $\mathrm{km}^{2}$ are expected. However, when the traffic density expected is greater or equal to 70.75 vehicles per $\mathrm{km}^{2}$, D-RSU is the best deployment policy. In addition, if there are more than 91 vehicles per $\mathrm{km}^{2}$, D-RSU obtains better results than the other approaches in terms of warning notification time, while reducing the required number of RSUs to a half.

According to the obtained results, we propose a RSU deployment algorithm which decides the optimum deployment policy based on the expected vehicle density. Since the density of vehicles is a time-varying factor, in the future we plan to make our approach adaptive, i.e., we consider to propose an adaptive RSU deployment algorithm capable of enable or disable the RSUs based on the density detected in that moment.

\section{ACKNOWLEDGMENTS}

This work was partially supported by the Ministerio de Educación y Ciencia, Spain, under Grant TIN2011-27543-C03-01.

\section{REFERENCES}

[1] J. Barrachina, P. Garrido, M. Fogue, F. J. Martinez, J.-C. Cano, C. T. Calafate, and P. Manzoni. VEACON: A Vehicular Accident Ontology designed to improve safety on the roads. Journal of Network and Computer Applications, 2012. Available at http://dx.doi.org/10.1016/j.jnca.2012.07.013.

[2] M. Fogue, P. Garrido, F. J. Martinez, J.-C. Cano, C. T. Calafate, and P. Manzoni. A Realistic Simulation Framework for Vehicular Networks. In 5th International ICST Conference on Simulation Tools and Techniques (SIMUTools 2012), Desenzano, Italy, pages 37-46, March 2012. Available at http://dx.doi.org/10.4108/icst.simutools.2012.247682.

[3] M. Fogue, P. Garrido, F. J. Martinez, J.-C. Cano, C. T. Calafate, and P. Manzoni. Evaluating the impact of a novel message dissemination scheme for vehicular networks using real maps. Transportation Research Part C: Emerging Technologies, 25:61-80, $2012 . \quad$ Available at http://dx.doi.org/10.1016/j.trc.2012.04.017.

[4] M. Fogue, P. Garrido, F. J. Martinez, J.-C. Cano, C. T. Calafate, P. Manzoni, and M. Sanchez. Prototyping an automatic notification scheme for traffic accidents in vehicular networks. In IFIP Wireless Days (WD), pages 1-5, Oct. 2011.

[5] A. Kchiche and F. Kamoun. Centrality-based access-points deployment for vehicular networks. In IEEE 17th International Conference on Telecommunications (ICT), pages 700-706, Apr. 2010.

[6] D. Krajzewicz, G. Hertkorn, C. Rossel, and P. Wagner. SUMO (Simulation of Urban MObility) - An open-source traffic simulation. In Proceedings of the 4th Middle East Symposium on Simulation and Modelling (MESM), pages 183-187, Sharjah, United Arab Emirates, Sep. 2002.

[7] J. Lee and C. Kim. A roadside unit placement scheme for vehicular telematics networks. In T.-H. Kim and H. Adeli, editors, Advances in Computer Science and Information Technology, volume 6059 of Lecture Notes in Computer Science, pages 196-202. Springer Berlin / Heidelberg, 2010.

[8] C. Lochert, B. Scheuermann, C. Wewetzer, A. Luebke, and M. Mauve. Data aggregation and roadside unit placement for a VANET traffic information system. In Proceedings of the fifth ACM international workshop on VehiculAr inter-NETworking, VANET '08, pages 58-65, New York, NY, USA, 2008. ACM.

[9] F. J. Martinez, J.-C. Cano, C. T. Calafate, and P. Manzoni. CityMob: a mobility model pattern generator for VANETs. In IEEE Vehicular Networks and Applications Workshop (Vehi-Mobi, held with ICC), Beijing, China, pages 370-374, May 2008. Available at http://dx.doi.org/10.1109/ICCW.2008.76.

[10] F. J. Martinez, M. Fogue, C. Toh, J.-C. Cano, C. T. Calafate, and P. Manzoni. Computer Simulations of VANETs Using Realistic City Topologies. Wireless Personal Communications Journal, 2012. Available at http://dx.doi.org/10.1007/s11277-012-0594-6.

[11] F. J. Martinez, C. K. Toh, J.-C. Cano, C. T. Calafate, and P. Manzoni. Emergency services in future intelligent transportation systems based on vehicular communication networks. IEEE Intelligent Transportation Systems Magazine, 2(2):6-20, summer 2010.

[12] K. Mershad, H. Artail, and M. Gerla. ROAMER: Roadside Units as message routers in VANETs. Ad Hoc Networks, 10(3):479-496, 2012.

[13] J. Santa, R. Toledo-Moreo, and A. F. Gomez-Skarmeta. A novel vehicle communication paradigm based on cellular networks for improving the safety in roads. In International Journal of Intelligent Information and Database Systems (IJIIDS), pages 240-257. INDERSCIENCE, 2008.

[14] J. Santa, R. Toledo-Moreo, M. A. Zamora-Izquierdo, B. beda Miarro, and A. F. Gmez-Skarmeta. An Analysis of Communication and Navigation Issues in Collision Avoidance Support Systems. In Transportation Research Part C. Emerging Technologies, pages 351-366. Elsevier, 2009. 
[15] Y.-C. Tseng, S.-Y. Ni, Y.-S. Chen, and J.-P. Sheu. The broadcast storm problem in a mobile ad hoc network. Wireless Networks, 8:153-167, 2002.

[16] T.-Y. Wu, S. Guizani, W.-T. Lee, and K.-H. Liao. Improving RSU service time by Distributed Sorting Mechanism. Ad Hoc Networks, 10(2):212-221, 2012 .

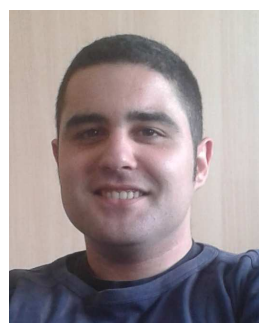

Javier Barrachina received his B.Sc. degree in Computer Science from the University of Zaragoza in 2011. Currently, he is a M.Sc. student from the National University of Distance Education (UNED), and a PhD candidate in the Intelligent Networks and Information Technologies (iNiT) research group. His research interests include VANET simulation, Intelligent Transportation Systems, virtual agents, ontologies, traffic safety, vehicle-to-vehicle (V2V), and vehicle-to-infrastructure (V2I) communications.

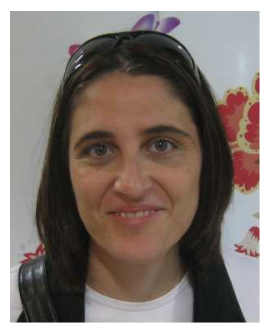

Piedad Garrido graduated in Computer Science and Documentation at the Technical University of Valencia in 1997 and 1999 , respectively. She received her Ph.D. degree in Documentation from the University Carlos III of Madrid in 2008. She is an assistant professor in the Department of Computers and Systems Engineering at the University of Zaragoza in Spain. Her current research interests include Intelligent Transportation Systems, virtual agents, ontologies, traffic safety, vehicle-to-vehicle (V2V), and vehicleto-infrastructure (V2I) communications. She is member of the IEEE.

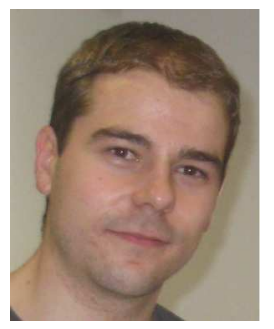

Manuel Fogue received his B.Sc. and M.Sc. degrees in Computer Science from the University of Zaragoza in 2007 and the University Jaume I of Castellon in 2009, respectively. In both cases, he graduated with honors. He received his Ph.D. degree in Computer Engineering from the University Zaragoza in 2012. He is a member of the Intelligent Networks and Information Technologies (iNiT) research group. His research interests include VANET simulation, Intelligent Transportation Systems, traffic safety, vehicle-to-vehicle (V2V), and vehicle-to-infrastructure (V2I) communications.

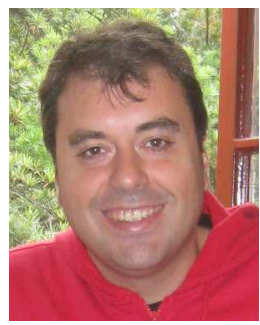

Francisco J. Martinez graduated in Computer Science and Documentation at the Technical University of Valencia in 1996 and 1999, respectively. He received his Ph.D. degree in Computer Engineering from the Technical University of Valencia in 2010, and received an Extraordinary Doctorate Award. He is an associate professor in the Department of Computers and Systems Engineering at the University of Zaragoza in Spain. He is a the leader of the Intelligent Networks and Information Technologies (iNiT) research group. His current research interests include VANET simulation, Intelligent Transportation Systems, traffic safety, vehicle-to-vehicle (V2V), and vehicle-to-infrastructure (V2I) communications. He is member of the IEEE.

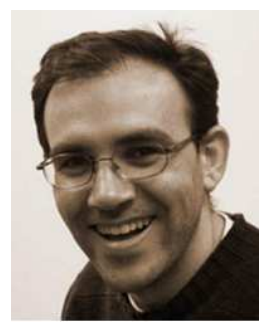

Juan-Carlos Cano received his M.Sc. and a Ph.D. in computer science from the Technical University of Valencia (UPV) in 1994 and 2002, respectively. From 1995 to 1997 he worked as a programming analyst at IBM's manufacturing division in Valencia. He is a full professor and the Head of the Department of Computer Engineering at the UPV in Spain. His current research interests include power aware routing protocols, quality of service for mobile ad hoc networks, and pervasive computing. 


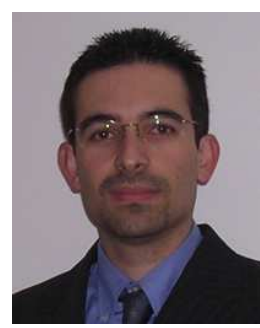

Carlos T. Calafate graduated with honors in Electrical and Computer Engineering at the University of Oporto (Portugal) in 2001. He received his Ph.D. degree in Computer Engineering from the Technical University of Valencia (UPV) in 2006, where he has worked since 2005. He is an associate professor in the Department of Computer Engineering at the UPV in Spain. He is a member of the Computer Networks research group (GRC). His research interests include mobile and pervasive computing, security and QoS on wireless networks, as well as video coding and streaming.

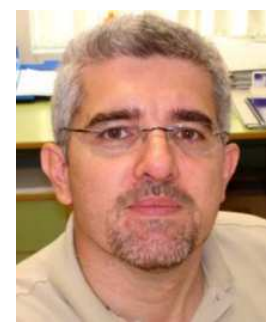

Pietro Manzoni received his MS degree in computer science from the "Universitá degli Studi" of Milan, Italy, in 1989, and the $\mathrm{Ph} . \mathrm{D}$. degree in computer science from the Polytechnic University of Milan, Italy, in 1995. He is a full professor in the Department of Computer Engineering at the Technical University of Valencia (UPV) in Spain. His research activity is related to wireless networks protocol design, modeling, and implementation. He is member of the IEEE. 